\title{
Scleroderma Renal Crisis: Still a Lot To Do
}

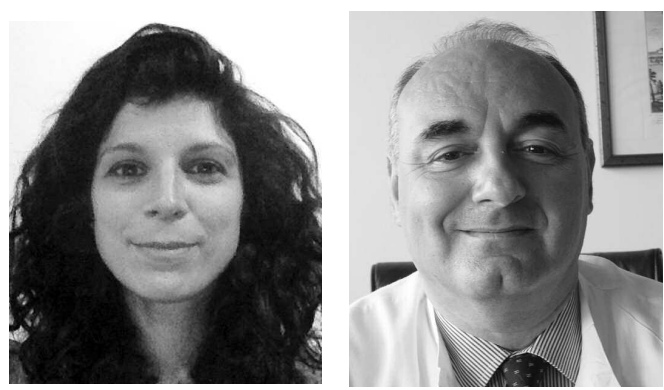

Scleroderma renal crisis (SRC) in recent literature history has been superseded by other major organ involvement (heart, lungs) as the leading cause of death in systemic sclerosis $(\mathrm{SSc})^{1}$. Despite this epidemiological shift, it remains one of the most serious and life-threatening complications regarding its individual effect on patients, with the added difficulty that research with randomized clinical or prospective studies is extremely difficult in its setting ${ }^{2}$.

Another issue concerns the definition of SRC, which is highly heterogeneous in the literature. A recent systematic literature review ${ }^{3}$ pointed out that practically every paper of the more than 40 included in the review used its own definition of SRC, both in the core characteristics (new-onset arterial hypertension, new and rapidly progressive acute kidney injury) and in the listing of the possible accompanying symptoms: hypertensive encephalopathy, seizures, thrombotic microangiopathy with anemia, and thrombocytopenia. There are diagnostic changes at the kidney biopsy, but there is no precise indication for it. Kidney biopsy appears necessary when differential diagnoses are not formally ruled out with only clinical data, but a successful procedure is complicated by the acute and severe clinical picture, especially when malignant hypertension, anemia, and thrombocytopenia, with consequent hemorrhagic risk, are present.

In this issue of The Journal, Gordon and colleagues ${ }^{4}$ retrospectively evaluated the US Department of Defense health care network in a valuable effort to identify subjects at risk for SRC in incident SSc cases. The strength of this report is a strict definition of SRC, especially at times when, as pointed out, criteria for SRC are lacking and are in the course of being developed ${ }^{3}$. The authors defined SRC, stressing the concept of acute kidney failure, eventually associated with malignant hypertension requiring hospitalization. Among their criteria, this last concept is a key point to their research and to the context in which this report should be read: the need for a strict definition encompassing hospitalization that strengthens the methods of a comprehensive electronic medical record review such as that reported in the manuscript. At the same time, though, this constitutes a considerable referral bias. To reduce the latter to the minimum, the authors decided to include in the analysis only patients with SSc without SRC at the onset, hence excluding those who presented SRC as the initial SSc manifestation. This has to be kept in mind when generalizing the results of the paper.

SRC is a manifestation prevalently occurring in the first phases of the disease course ( $<4 \mathrm{yrs})$, and there are specific populations of patients identified by other clinical risk factors to take into account such as older age, an African American ethnicity, corticosteroid use, diffuse cutaneous involvement, anti-RNA polymerase III (anti-RNAPIII) antibody positivity, and other musculoskeletal inflammatory complications such as arthralgias, synovitis, and tendon friction rubs ${ }^{5,6,7,8}$. These subjects should be persistently considered at risk for SRC even after the first years of disease course and especially if exposed to steroid therapy with medium to high doses. A further important precipitating factor in SRC that emerged with the analysis by Gordon and colleagues ${ }^{4}$ is the contemporary (synchronous) or slightly delayed concomitant diagnosis of a malignancy. As recently reported $^{9}$ in a European League Against Rheumatism Scleroderma Trials and Research group survey and a case control study, there could be an indirect link between anti-RNAPIII antibody positivity, SRC, and oncogenesis owing to the association between this autoantibody subset and the increased risk of both clinical manifestations. A careful screening for cancer is recommended at the moment for anti-RNAPIII antibody-positive subjects ${ }^{9}$. There also should be discussion about the implications/modifications for the subsequent treatment strategies for cancer, with the specific aim to prevent SRC in this particular subset of patients.

So far, kidney-related risk factors such as reduced glomerular filtration rate, increased renal resistive index, isolated proteinuria, or isolated hypertension were not shown to specifically predict future SRC development ${ }^{10}$. In the

See Scleroderma renal crisis risk factors, page 85

Personal non-commercial use only. The Journal of Rheumatology Copyright (๑) 2019. All rights reserved. 
report by Gordon, et $a l^{4}$, the combination of $\geq 2$ risk factors was highly sensitive (95\%), and of $\geq 4$ at SSc diagnosis specific (65\%) for the prediction of SRC development in the following 3 years. This is the first report, to our knowledge, to link the association of multiple kidney variables to the short-term followup of a large cohort of patients. This is also a retrospective evaluation and there could be some circularity in the definition given for the diagnosis of SRC and the kidney-specific variables that are found significantly associated with the crisis, which are considered risk factors but are also key components of the acute clinical picture and of its diagnosis. It is difficult, given the low number of events and the retrospective nature of the analysis, to draw any further conclusion at the moment.

Finally, it is crucial, starting with a precise and agreed-upon definition of SRC and its diagnosis, to analyze cohorts longitudinally and thoroughly, given the relatively low cost of a specific kidney followup, and on the other side, the extremely high cost of the clinical picture and its complicated therapy, unfortunately still unsuccessful in an unacceptably high number of patients.

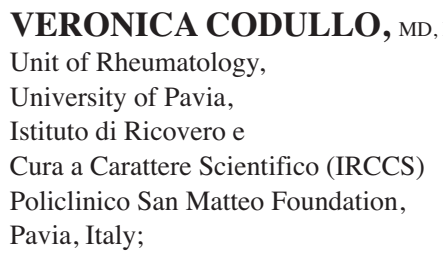

CARLOMAURIZIO MONTECUCCO, MD, Full Professor of Rheumatology, Unit of Rheumatology,

University of Pavia,

IRCCS Policlinico San Matteo Foundation, Pavia, Italy.

Address correspondence to Prof. C. Montecucco, Unit of Rheumatology, University of Pavia, IRCCS Policlinico San Matteo Foundation, Piazzale Golgi 19, 27100 Pavia, Italy. E-mail: montecucco@smatteo.pv.it

\section{REFERENCES}

1. Elhai M, Meune C, Boubaya M, Avouac J, Hachulla E, Balbir-Gurman A, et al. Mapping and predicting mortality from systemic sclerosis. Ann Rheum Dis 2017;76:1897-905.

2. Kowal-Bielecka O, Fransen J, Avouac J, Becker M, Kulak A, Allanore Y, et al. Update of EULAR recommendations for the treatment of systemic sclerosis. Ann Rheum Dis 2017;76:1327-39.

3. Hoa S, Stern EP, Denton CP, Hudson M. Towards developing criteria for scleroderma renal crisis: A scoping review. Autoimmun Rev 2017;16:407-15.

4. Gordon S, Edison J, Nee R, Stitt R, Bailey W, Little D, et al. Risk factors for future scleroderma renal crisis at systemic sclerosis diagnosis. J Rheumatol 2019;46;85-92.

5. Guillevin L, Bérezné A, Seror R, Teixeira L, Pourrat J, Mahr A, et al. Scleroderma renal crisis: a retrospective multicentre study on 91 patients and 427 controls. Rheumatology 2012;51:460-7.

6. Doré A, Lucas M, Ivanco D, Medsger TA, Domsic RT. Significance of palpable tendon friction rubs in early diffuse cutaneous systemic sclerosis. Arthritis Care Res 2013;65:1385-9.

7. Avouac J, Walker UA, Hachulla E, Riemekasten G, Cuomo G, Carreira PE, et al. Joint and tendon involvement predict disease progression in systemic sclerosis: a EUSTAR prospective study. Ann Rheum Dis 2016;75:103-9.

8. Hudson M, Baron M, Tatibouet S, Furst DE, Khanna D; International Scleroderma Renal Crisis Study Investigators. Exposure to ACE inhibitors prior to the onset of scleroderma renal crisis-results from the International Scleroderma Renal Crisis Survey. Semin Arthritis Rheum 2014;43:666-72.

9. Lazzaroni MG, Cavazzana I, Colombo E, Dobrota R, Hernandez J, Hesselstrand R, et al. Malignancies in patients with anti-RNA polymerase III antibodies and systemic sclerosis: analysis of the EULAR Scleroderma Trials and Research Cohort and possible recommendations for screening. J Rheumatol 2017;44:639-47.

10. Woodworth TG, Suliman YA, Li W, Furst DE, Clements P. Scleroderma renal crisis and renal involvement in systemic sclerosis. Nat Rev Nephrol 2018;14:137.

J Rheumatol 2019;46:3-4; doi:10.3899/jrheum.180594

Personal non-commercial use only. The Journal of Rheumatology Copyright (c) 2019. All rights reserved. 